Supporting Information

\title{
Strategy to Manipulate Molecular Orientation and Charge Mobility in D-A Type Conjugated Polymer through Rational Fluorination for Improvements of Photovoltaic Performances
}

Meng Qiu, ${ }^{\dagger}$ Dangqiang Zhu,${ }^{\dagger}$ Linyin Yan,${ }^{\S}$ Ning Wang, ${ }^{\dagger}$ Liangliang Han, ${ }^{\dagger}$ Xichang Bao, ${ }^{\dagger}$ Zurong Du, ${ }^{\dagger}$ Yingli Niu $*^{*}$ and Renqiang Yang* ${ }^{\dagger}$

${ }^{\dagger}$ CAS Key Laboratory of Bio-based Materials, Qingdao Institute of Bioenergy and Bioprocess Technology, Chinese Academy of Sciences, Qingdao 266101, China.

* Key Laboratory of Standardization and Measurement for Nanotechnology, Chinese Academy of Sciences, National Center for Nanoscience and Technology, Beijing 100190, China.

$\S$ The National Key Laboratory of Biochemical Engineering Institute of Process Engineering, Chinese Academy of Sciences, Zhongguancun North Second Street 1, Beijing 100190, China. 


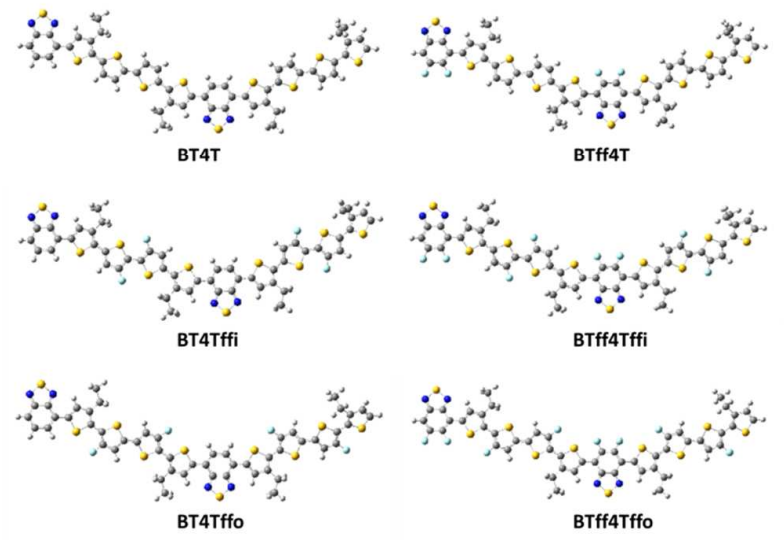

Figure S1. Optimized structures of BT4T and its fluorinated derivatives (grey: C, white: H, yellow: S, light blue: F, blue: N).

Table S1. The optimized molecular coordinates of BT4T and its fluorinated derivatives

\begin{tabular}{|c|c|c|c|c|c|c|c|}
\hline atom & $\mathrm{x}(\AA)$ & $\mathrm{y}(\AA)$ & $\mathrm{z}(\AA)$ & atom & $\mathrm{x}(\AA)$ & $\mathrm{y}(\AA)$ & $\mathrm{z}(\AA)$ \\
\hline \multicolumn{4}{|c|}{ BT4T } & \multicolumn{4}{|c|}{ BTff4T } \\
\hline $\mathrm{C}$ & -17.725 & -2.435 & -0.118 & $\mathrm{C}$ & -17.445 & -2.449 & -0.035 \\
\hline $\mathrm{C}$ & -16.274 & -2.559 & -0.080 & $\mathrm{C}$ & -15.990 & -2.548 & -0.007 \\
\hline $\mathrm{C}$ & -15.439 & -1.382 & 0.035 & $\mathrm{C}$ & -15.153 & -1.360 & 0.008 \\
\hline $\mathrm{C}$ & -16.119 & -0.175 & 0.110 & $\mathrm{C}$ & -15.869 & -0.177 & -0.005 \\
\hline $\mathrm{C}$ & -17.537 & -0.065 & 0.070 & $\mathrm{C}$ & -17.293 & -0.091 & -0.032 \\
\hline $\mathrm{C}$ & -18.352 & -1.161 & -0.041 & $\mathrm{C}$ & -18.101 & -1.187 & -0.048 \\
\hline $\mathrm{H}$ & -19.433 & -1.089 & -0.070 & $\mathrm{H}$ & -19.180 & -1.103 & -0.068 \\
\hline $\mathrm{C}$ & -13.985 & -1.459 & 0.069 & $\mathrm{C}$ & -13.699 & -1.417 & 0.035 \\
\hline $\mathrm{C}$ & -13.166 & -2.569 & 0.114 & $\mathrm{C}$ & -12.896 & -2.543 & 0.056 \\
\hline $\mathrm{S}$ & -13.005 & -0.003 & 0.051 & $\mathrm{~S}$ & -12.690 & 0.022 & 0.036 \\
\hline $\mathrm{C}$ & -11.773 & -2.292 & 0.151 & $\mathrm{C}$ & -11.502 & -2.292 & 0.083 \\
\hline $\mathrm{H}$ & -13.576 & -3.568 & 0.126 & $\mathrm{H}$ & -13.320 & -3.535 & 0.060 \\
\hline $\mathrm{C}$ & -11.510 & -0.924 & 0.137 & $\mathrm{C}$ & -11.218 & -0.926 & 0.087 \\
\hline $\mathrm{C}$ & -10.266 & -0.189 & 0.181 & $\mathrm{C}$ & -9.959 & -0.216 & 0.134 \\
\hline $\mathrm{C}$ & -10.096 & 1.161 & 0.440 & $\mathrm{C}$ & -9.762 & 1.127 & 0.407 \\
\hline $\mathrm{S}$ & -8.700 & -0.923 & -0.155 & $\mathrm{~S}$ & -8.408 & -0.977 & -0.212 \\
\hline $\mathrm{C}$ & -8.757 & 1.600 & 0.373 & $\mathrm{C}$ & -8.416 & 1.541 & 0.339 \\
\hline $\mathrm{H}$ & -10.923 & 1.816 & 0.687 & $\mathrm{H}$ & -10.576 & 1.796 & 0.662 \\
\hline $\mathrm{C}$ & -7.855 & 0.599 & 0.062 & $\mathrm{C}$ & -7.535 & 0.526 & 0.013 \\
\hline $\mathrm{H}$ & -8.458 & 2.625 & 0.559 & $\mathrm{H}$ & -8.097 & 2.560 & 0.529 \\
\hline $\mathrm{C}$ & -6.422 & 0.673 & -0.072 & $\mathrm{C}$ & -6.101 & 0.575 & -0.128 \\
\hline $\mathrm{C}$ & -5.524 & -0.324 & -0.404 & $\mathrm{C}$ & -5.229 & -0.423 & -0.522 \\
\hline $\mathrm{S}$ & -5.571 & 2.188 & 0.176 & $\mathrm{~S}$ & -5.213 & 2.052 & 0.200 \\
\hline $\mathrm{C}$ & -4.184 & 0.109 & -0.462 & $\mathrm{C}$ & -3.880 & -0.017 & -0.567 \\
\hline
\end{tabular}




\begin{tabular}{|c|c|c|c|c|c|c|c|}
\hline $\mathrm{H}$ & -5.828 & -1.344 & -0.612 & $\mathrm{H}$ & -5.559 & -1.421 & -0.788 \\
\hline $\mathrm{C}$ & -4.008 & 1.454 & -0.177 & $\mathrm{C}$ & -3.670 & 1.304 & -0.207 \\
\hline $\mathrm{H}$ & -3.360 & -0.544 & -0.723 & $\mathrm{H}$ & -3.074 & -0.674 & -0.872 \\
\hline $\mathrm{C}$ & -2.761 & 2.181 & -0.121 & $\mathrm{C}$ & -2.405 & 1.996 & -0.121 \\
\hline $\mathrm{C}$ & -2.488 & 3.547 & -0.083 & $\mathrm{C}$ & -2.104 & 3.355 & -0.027 \\
\hline $\mathrm{S}$ & -1.269 & 1.250 & -0.099 & $\mathrm{~S}$ & -0.943 & 1.028 & -0.141 \\
\hline $\mathrm{C}$ & -1.095 & 3.816 & -0.055 & $\mathrm{C}$ & -0.707 & 3.589 & 0.008 \\
\hline $\mathrm{C}$ & -0.280 & 2.701 & -0.070 & $\mathrm{C}$ & 0.083 & 2.453 & -0.049 \\
\hline $\mathrm{H}$ & -0.680 & 4.812 & -0.032 & $\mathrm{H}$ & -0.272 & 4.574 & 0.068 \\
\hline $\mathrm{N}$ & -18.363 & -3.607 & -0.230 & $\mathrm{~N}$ & -18.065 & -3.631 & -0.048 \\
\hline $\mathrm{N}$ & -15.856 & -3.826 & -0.166 & $\mathrm{~N}$ & -15.558 & -3.810 & -0.000 \\
\hline $\mathrm{S}$ & -17.201 & -4.766 & -0.282 & $\mathrm{~S}$ & -16.889 & -4.778 & -0.026 \\
\hline $\mathrm{C}$ & 1.168 & 2.610 & -0.061 & $\mathrm{~F}$ & -17.806 & 1.145 & -0.042 \\
\hline $\mathrm{C}$ & 2.026 & 3.765 & 0.070 & $\mathrm{~F}$ & -15.231 & 1.008 & 0.004 \\
\hline $\mathrm{C}$ & 1.841 & 1.400 & -0.179 & $\mathrm{C}$ & 1.532 & 2.373 & -0.034 \\
\hline $\mathrm{C}$ & 3.482 & 3.634 & 0.082 & $\mathrm{C}$ & 2.399 & 3.529 & 0.066 \\
\hline $\mathrm{N}$ & 1.614 & 5.029 & 0.196 & $\mathrm{C}$ & 2.238 & 1.185 & -0.113 \\
\hline $\mathrm{C}$ & 3.245 & 1.274 & -0.169 & $\mathrm{C}$ & 3.859 & 3.413 & 0.078 \\
\hline $\mathrm{C}$ & 4.122 & 2.345 & -0.040 & $\mathrm{~N}$ & 1.979 & 4.790 & 0.157 \\
\hline $\mathrm{N}$ & 4.110 & 4.805 & 0.215 & $\mathrm{C}$ & 3.649 & 1.073 & -0.102 \\
\hline S & 2.956 & 5.968 & 0.317 & $\mathrm{~F}$ & 1.584 & 0.013 & -0.208 \\
\hline $\mathrm{C}$ & 5.564 & 2.175 & -0.030 & $\mathrm{C}$ & 4.533 & 2.133 & -0.011 \\
\hline $\mathrm{C}$ & 6.565 & 3.125 & 0.033 & $\mathrm{~N}$ & 4.472 & 4.591 & 0.178 \\
\hline S & 6.278 & 0.572 & -0.104 & $\mathrm{~S}$ & 3.309 & 5.749 & 0.250 \\
\hline $\mathrm{C}$ & 7.888 & 2.611 & 0.010 & $\mathrm{~F}$ & 4.110 & -0.187 & -0.192 \\
\hline $\mathrm{H}$ & 6.335 & 4.178 & 0.088 & $\mathrm{C}$ & 5.976 & 1.982 & -0.006 \\
\hline $\mathrm{C}$ & 7.913 & 1.220 & -0.076 & $\mathrm{C}$ & 6.937 & 2.977 & 0.082 \\
\hline $\mathrm{C}$ & 9.011 & 0.285 & -0.149 & $\mathrm{~S}$ & 6.766 & 0.414 & -0.112 \\
\hline $\mathrm{C}$ & 8.949 & -1.059 & -0.479 & $\mathrm{C}$ & 8.279 & 2.525 & 0.056 \\
\hline S & 10.677 & 0.717 & 0.231 & $\mathrm{H}$ & 6.662 & 4.017 & 0.155 \\
\hline $\mathrm{C}$ & 10.190 & -1.728 & -0.424 & $\mathrm{C}$ & 8.362 & 1.138 & -0.060 \\
\hline $\mathrm{H}$ & 8.025 & -1.548 & -0.763 & $\mathrm{C}$ & 9.504 & 0.256 & -0.152 \\
\hline $\mathrm{C}$ & 11.245 & -0.917 & -0.050 & $\mathrm{C}$ & 9.509 & -1.072 & -0.543 \\
\hline $\mathrm{H}$ & 10.310 & -2.781 & -0.649 & $\mathrm{~S}$ & 11.141 & 0.743 & 0.284 \\
\hline $\mathrm{C}$ & 12.643 & -1.249 & 0.094 & $\mathrm{C}$ & 10.777 & -1.689 & -0.489 \\
\hline $\mathrm{C}$ & 13.658 & -0.513 & 0.673 & $\mathrm{H}$ & 8.613 & -1.587 & -0.870 \\
\hline $\mathrm{S}$ & 13.272 & -2.778 & -0.498 & $\mathrm{C}$ & 11.787 & -0.850 & -0.057 \\
\hline $\mathrm{C}$ & 14.914 & -1.167 & 0.663 & $\mathrm{H}$ & 10.948 & -2.725 & -0.759 \\
\hline $\mathrm{H}$ & 13.496 & 0.460 & 1.120 & $\mathrm{C}$ & 13.194 & -1.129 & 0.105 \\
\hline $\mathrm{C}$ & 14.891 & -2.419 & 0.084 & $\mathrm{C}$ & 14.168 & -0.369 & 0.722 \\
\hline $\mathrm{H}$ & 15.815 & -0.744 & 1.093 & $\mathrm{~S}$ & 13.895 & -2.614 & -0.515 \\
\hline $\mathrm{C}$ & 16.016 & -3.316 & -0.141 & $\mathrm{C}$ & 15.449 & -0.971 & 0.720 \\
\hline $\mathrm{C}$ & 16.137 & -4.688 & -0.000 & $\mathrm{H}$ & 13.958 & 0.585 & 1.191 \\
\hline $\mathrm{S}$ & 17.528 & -2.606 & -0.699 & $\mathrm{C}$ & 15.488 & -2.207 & 0.108 \\
\hline
\end{tabular}




\begin{tabular}{|c|c|c|c|c|c|c|c|}
\hline $\mathrm{C}$ & 17.452 & -5.145 & -0.339 & $\mathrm{H}$ & 16.324 & -0.525 & 1.178 \\
\hline $\mathrm{C}$ & 18.306 & -4.150 & -0.720 & $\mathrm{C}$ & 16.652 & -3.053 & -0.117 \\
\hline $\mathrm{H}$ & 17.745 & -6.188 & -0.290 & $\mathrm{C}$ & 16.824 & -4.422 & -0.009 \\
\hline $\mathrm{H}$ & 19.342 & -4.234 & -1.017 & $\mathrm{~S}$ & 18.144 & -2.269 & -0.626 \\
\hline $\mathrm{C}$ & 9.121 & 3.489 & 0.061 & $\mathrm{C}$ & 18.163 & -4.818 & -0.333 \\
\hline $\mathrm{H}$ & 9.663 & 3.296 & 0.997 & $\mathrm{C}$ & 18.984 & -3.781 & -0.671 \\
\hline $\mathrm{H}$ & 9.807 & 3.190 & -0.740 & $\mathrm{H}$ & 18.496 & -5.850 & -0.304 \\
\hline $\mathrm{C}$ & 8.854 & 4.995 & -0.048 & $\mathrm{H}$ & 20.028 & -3.816 & -0.949 \\
\hline $\mathrm{H}$ & 8.338 & 5.244 & -0.980 & $\mathrm{C}$ & 9.470 & 3.456 & 0.132 \\
\hline $\mathrm{H}$ & 8.245 & 5.361 & 0.783 & $\mathrm{H}$ & 10.011 & 3.276 & 1.071 \\
\hline $\mathrm{H}$ & 9.800 & 5.544 & -0.032 & $\mathrm{H}$ & 10.178 & 3.198 & -0.665 \\
\hline $\mathrm{C}$ & 15.072 & -5.629 & 0.512 & $\mathrm{C}$ & 9.138 & 4.950 & 0.037 \\
\hline $\mathrm{H}$ & 14.126 & -5.095 & 0.636 & $\mathrm{H}$ & 8.620 & 5.187 & -0.896 \\
\hline $\mathrm{H}$ & 14.888 & -6.408 & -0.239 & $\mathrm{H}$ & 8.506 & 5.279 & 0.867 \\
\hline $\mathrm{C}$ & 15.450 & -6.295 & 1.847 & $\mathrm{H}$ & 10.059 & 5.540 & 0.068 \\
\hline $\mathrm{H}$ & 16.375 & -6.874 & 1.760 & $\mathrm{C}$ & 15.787 & -5.417 & 0.456 \\
\hline $\mathrm{H}$ & 15.600 & -5.544 & 2.628 & $\mathrm{H}$ & 14.820 & -4.924 & 0.577 \\
\hline $\mathrm{H}$ & 14.658 & -6.976 & 2.175 & $\mathrm{H}$ & 15.647 & -6.180 & -0.320 \\
\hline $\mathrm{C}$ & -3.543 & 4.634 & -0.084 & $\mathrm{C}$ & 16.167 & -6.108 & 1.778 \\
\hline $\mathrm{H}$ & -4.270 & 4.427 & -0.879 & $\mathrm{H}$ & 17.115 & -6.649 & 1.692 \\
\hline $\mathrm{H}$ & -4.113 & 4.585 & 0.853 & $\mathrm{H}$ & 16.274 & -5.375 & 2.584 \\
\hline $\mathrm{C}$ & -3.009 & 6.060 & -0.258 & $\mathrm{H}$ & 15.396 & -6.828 & 2.071 \\
\hline $\mathrm{H}$ & -2.346 & 6.347 & 0.563 & $\mathrm{C}$ & -3.132 & 4.466 & 0.016 \\
\hline $\mathrm{H}$ & -2.452 & 6.168 & -1.194 & $\mathrm{H}$ & -3.867 & 4.305 & -0.781 \\
\hline $\mathrm{H}$ & -3.839 & 6.771 & -0.278 & $\mathrm{H}$ & -3.700 & 4.396 & 0.955 \\
\hline $\mathrm{C}$ & -10.714 & -3.372 & 0.215 & $\mathrm{C}$ & -2.565 & 5.884 & -0.107 \\
\hline $\mathrm{H}$ & -10.138 & -3.369 & -0.719 & $\mathrm{H}$ & -1.894 & 6.127 & 0.722 \\
\hline $\mathrm{H}$ & -9.993 & -3.120 & 1.003 & $\mathrm{H}$ & -2.007 & 6.013 & -1.040 \\
\hline $\mathrm{C}$ & -11.243 & -4.790 & 0.460 & $\mathrm{H}$ & -3.378 & 6.615 & -0.099 \\
\hline $\mathrm{H}$ & -11.806 & -4.852 & 1.396 & $\mathrm{C}$ & -10.462 & -3.392 & 0.117 \\
\hline $\mathrm{H}$ & -11.898 & -5.124 & -0.350 & $\mathrm{H}$ & -9.885 & -3.372 & -0.817 \\
\hline $\mathrm{H}$ & -10.409 & -5.494 & 0.523 & $\mathrm{H}$ & -9.737 & -3.175 & 0.912 \\
\hline $\mathrm{H}$ & -17.974 & 0.926 & 0.135 & $\mathrm{C}$ & -11.015 & -4.806 & 0.321 \\
\hline $\mathrm{H}$ & -15.549 & 0.742 & 0.210 & $\mathrm{H}$ & -11.578 & -4.886 & 1.257 \\
\hline $\mathrm{H}$ & 1.266 & 0.487 & -0.294 & $\mathrm{H}$ & -11.677 & -5.105 & -0.496 \\
\hline $\mathrm{H}$ & 3.650 & 0.272 & -0.275 & $\mathrm{H}$ & -10.194 & -5.527 & 0.362 \\
\hline \multicolumn{4}{|c|}{ BT4Tffi } & \multicolumn{4}{|c|}{ BTff4Tffi } \\
\hline $\mathrm{C}$ & -17.879 & -2.487 & -0.139 & $\mathrm{C}$ & -17.639 & -2.443 & -0.101 \\
\hline $\mathrm{C}$ & -16.428 & -2.605 & -0.095 & $\mathrm{C}$ & -16.185 & -2.547 & -0.061 \\
\hline $\mathrm{C}$ & -15.598 & -1.423 & -0.010 & $\mathrm{C}$ & -15.344 & -1.362 & -0.039 \\
\hline $\mathrm{C}$ & -16.281 & -0.217 & 0.028 & $\mathrm{C}$ & -16.056 & -0.176 & -0.058 \\
\hline $\mathrm{C}$ & -17.699 & -0.111 & -0.015 & $\mathrm{C}$ & -17.479 & -0.085 & -0.097 \\
\hline $\mathrm{C}$ & -18.510 & -1.213 & -0.098 & $\mathrm{C}$ & -18.291 & -1.179 & -0.118 \\
\hline $\mathrm{H}$ & -19.591 & -1.145 & -0.131 & $\mathrm{H}$ & -19.369 & -1.092 & -0.148 \\
\hline
\end{tabular}




\begin{tabular}{|c|c|c|c|c|c|c|c|}
\hline $\mathrm{C}$ & -14.143 & -1.495 & 0.029 & $\mathrm{C}$ & -13.891 & -1.424 & -0.000 \\
\hline $\mathrm{C}$ & -13.320 & -2.602 & 0.077 & $\mathrm{C}$ & -13.092 & -2.553 & 0.023 \\
\hline S & -13.169 & -0.035 & 0.013 & S & -12.878 & 0.012 & 0.013 \\
\hline $\mathrm{C}$ & -11.929 & -2.320 & 0.115 & $\mathrm{C}$ & -11.697 & -2.307 & 0.058 \\
\hline $\mathrm{H}$ & -13.727 & -3.602 & 0.089 & $\mathrm{H}$ & -13.519 & -3.543 & 0.019 \\
\hline $\mathrm{C}$ & -11.670 & -0.951 & 0.096 & $\mathrm{C}$ & -11.409 & -0.941 & 0.065 \\
\hline $\mathrm{C}$ & -10.429 & -0.213 & 0.136 & $\mathrm{C}$ & -10.148 & -0.237 & 0.113 \\
\hline $\mathrm{C}$ & -10.276 & 1.152 & 0.306 & $\mathrm{C}$ & -9.962 & 1.124 & 0.285 \\
\hline S & -8.853 & -0.977 & -0.075 & S & -8.591 & -1.042 & -0.085 \\
\hline $\mathrm{C}$ & -8.931 & 1.552 & 0.263 & $\mathrm{C}$ & -8.607 & 1.489 & 0.254 \\
\hline $\mathrm{H}$ & -11.091 & 1.847 & 0.466 & $\mathrm{H}$ & -10.758 & 1.840 & 0.437 \\
\hline $\mathrm{C}$ & -8.000 & 0.552 & 0.066 & $\mathrm{C}$ & -7.700 & 0.464 & 0.064 \\
\hline $\mathrm{C}$ & -6.571 & 0.661 & -0.003 & $\mathrm{C}$ & -6.269 & 0.536 & 0.004 \\
\hline $\mathrm{C}$ & -5.642 & -0.340 & -0.201 & $\mathrm{C}$ & -5.364 & -0.489 & -0.184 \\
\hline S & -5.716 & 2.189 & 0.145 & S & -5.376 & 2.042 & 0.158 \\
\hline $\mathrm{C}$ & -4.296 & 0.056 & -0.238 & $\mathrm{C}$ & -4.008 & -0.126 & -0.212 \\
\hline $\mathrm{C}$ & -4.140 & 1.422 & -0.064 & $\mathrm{C}$ & -3.819 & 1.234 & -0.038 \\
\hline $\mathrm{H}$ & -3.484 & -0.640 & -0.395 & $\mathrm{H}$ & -3.213 & -0.845 & -0.361 \\
\hline $\mathrm{C}$ & -2.898 & 2.155 & -0.028 & $\mathrm{C}$ & -2.557 & 1.935 & 0.002 \\
\hline $\mathrm{C}$ & -2.633 & 3.523 & 0.023 & $\mathrm{C}$ & -2.264 & 3.298 & 0.063 \\
\hline S & -1.399 & 1.234 & -0.054 & S & -1.088 & 0.977 & -0.031 \\
\hline $\mathrm{C}$ & -1.242 & 3.801 & 0.031 & $\mathrm{C}$ & -0.869 & 3.542 & 0.072 \\
\hline $\mathrm{C}$ & -0.420 & 2.691 & -0.012 & $\mathrm{C}$ & -0.071 & 2.411 & 0.023 \\
\hline $\mathrm{H}$ & -0.833 & 4.799 & 0.064 & $\mathrm{H}$ & -0.440 & 4.530 & 0.111 \\
\hline $\mathrm{N}$ & -18.513 & -3.663 & -0.219 & $\mathrm{~N}$ & -18.263 & -3.623 & -0.118 \\
\hline $\mathrm{N}$ & -16.006 & -3.873 & -0.145 & $\mathrm{~N}$ & -15.758 & -3.810 & -0.049 \\
\hline S & -17.348 & -4.820 & -0.238 & $\mathrm{~S}$ & -17.091 & -4.774 & -0.085 \\
\hline $\mathrm{C}$ & 1.029 & 2.610 & -0.025 & $\mathrm{C}$ & 1.377 & 2.341 & 0.017 \\
\hline $\mathrm{C}$ & 1.880 & 3.772 & 0.081 & $\mathrm{C}$ & 2.237 & 3.506 & 0.072 \\
\hline $\mathrm{C}$ & 1.708 & 1.403 & -0.141 & $\mathrm{C}$ & 2.090 & 1.157 & -0.042 \\
\hline $\mathrm{C}$ & 3.337 & 3.651 & 0.069 & $\mathrm{C}$ & 3.697 & 3.401 & 0.061 \\
\hline $\mathrm{N}$ & 1.462 & 5.035 & 0.200 & $\mathrm{~N}$ & 1.808 & 4.766 & 0.138 \\
\hline $\mathrm{C}$ & 3.112 & 1.287 & -0.153 & $\mathrm{C}$ & 3.502 & 1.055 & -0.054 \\
\hline $\mathrm{C}$ & 3.983 & 2.365 & -0.050 & $\mathrm{C}$ & 4.379 & 2.124 & -0.007 \\
\hline $\mathrm{N}$ & 3.959 & 4.828 & 0.180 & $\mathrm{~N}$ & 4.303 & 4.587 & 0.118 \\
\hline S & 2.799 & 5.984 & 0.289 & S & 3.132 & 5.736 & 0.182 \\
\hline $\mathrm{C}$ & 5.426 & 2.205 & -0.063 & $\mathrm{C}$ & 5.823 & 1.985 & -0.025 \\
\hline $\mathrm{C}$ & 6.422 & 3.162 & -0.009 & $\mathrm{C}$ & 6.777 & 2.989 & 0.020 \\
\hline S & 6.150 & 0.607 & -0.152 & S & 6.624 & 0.421 & -0.109 \\
\hline $\mathrm{C}$ & 7.748 & 2.658 & -0.048 & $\mathrm{C}$ & 8.121 & 2.549 & -0.017 \\
\hline $\mathrm{H}$ & 6.185 & 4.213 & 0.052 & $\mathrm{H}$ & 6.494 & 4.029 & 0.075 \\
\hline $\mathrm{C}$ & 7.780 & 1.267 & -0.137 & $\mathrm{C}$ & 8.215 & 1.158 & -0.099 \\
\hline $\mathrm{C}$ & 8.883 & 0.340 & -0.221 & $\mathrm{C}$ & 9.362 & 0.285 & -0.184 \\
\hline $\mathrm{C}$ & 8.813 & -1.014 & -0.499 & $\mathrm{C}$ & 9.356 & -1.071 & -0.458 \\
\hline
\end{tabular}




\begin{tabular}{|c|c|c|c|c|c|c|c|}
\hline $\mathrm{S}$ & 10.558 & 0.812 & 0.072 & $\mathrm{~S}$ & 11.014 & 0.840 & 0.095 \\
\hline $\mathrm{C}$ & 10.073 & -1.634 & -0.473 & $\mathrm{C}$ & 10.645 & -1.629 & -0.440 \\
\hline $\mathrm{H}$ & 7.900 & -1.550 & -0.725 & $\mathrm{H}$ & 8.469 & -1.652 & -0.677 \\
\hline $\mathrm{C}$ & 11.150 & -0.821 & -0.185 & $\mathrm{C}$ & 11.682 & -0.763 & -0.161 \\
\hline $\mathrm{C}$ & 12.541 & -1.172 & -0.109 & $\mathrm{C}$ & 13.090 & -1.045 & -0.096 \\
\hline $\mathrm{C}$ & 13.618 & -0.359 & 0.172 & $\mathrm{C}$ & 14.127 & -0.179 & 0.176 \\
\hline $\mathrm{S}$ & 13.136 & -2.809 & -0.358 & $\mathrm{~S}$ & 13.763 & -2.650 & -0.354 \\
\hline $\mathrm{C}$ & 14.881 & -0.985 & 0.214 & $\mathrm{C}$ & 15.420 & -0.741 & 0.204 \\
\hline $\mathrm{C}$ & 14.800 & -2.337 & -0.039 & $\mathrm{C}$ & 15.405 & -2.095 & -0.051 \\
\hline $\mathrm{H}$ & 15.799 & -0.460 & 0.442 & $\mathrm{H}$ & 16.313 & -0.171 & 0.424 \\
\hline $\mathrm{C}$ & 15.903 & -3.281 & -0.149 & $\mathrm{C}$ & 16.553 & -2.981 & -0.175 \\
\hline $\mathrm{C}$ & 16.040 & -4.585 & 0.296 & $\mathrm{C}$ & 16.761 & -4.277 & 0.265 \\
\hline $\mathrm{S}$ & 17.358 & -2.744 & -0.983 & $\mathrm{~S}$ & 17.970 & -2.368 & -1.022 \\
\hline $\mathrm{C}$ & 17.322 & -5.129 & -0.038 & $\mathrm{C}$ & 18.067 & -4.754 & -0.083 \\
\hline $\mathrm{C}$ & 18.135 & -4.265 & -0.713 & $\mathrm{C}$ & 18.827 & -3.848 & -0.763 \\
\hline $\mathrm{H}$ & 17.622 & -6.137 & 0.226 & $\mathrm{H}$ & 18.421 & -5.746 & 0.175 \\
\hline $\mathrm{H}$ & 19.141 & -4.434 & -1.071 & $\mathrm{H}$ & 19.837 & -3.964 & -1.132 \\
\hline $\mathrm{C}$ & 8.974 & 3.545 & -0.007 & $\mathrm{C}$ & 9.304 & 3.493 & 0.015 \\
\hline $\mathrm{H}$ & 9.542 & 3.333 & 0.909 & $\mathrm{H}$ & 9.887 & 3.309 & 0.929 \\
\hline $\mathrm{H}$ & 9.642 & 3.271 & -0.834 & $\mathrm{H}$ & 9.979 & 3.250 & -0.814 \\
\hline $\mathrm{C}$ & 8.694 & 5.050 & -0.072 & $\mathrm{C}$ & 8.954 & 4.983 & -0.049 \\
\hline $\mathrm{H}$ & 8.157 & 5.320 & -0.987 & $\mathrm{H}$ & 8.399 & 5.226 & -0.960 \\
\hline $\mathrm{H}$ & 8.100 & 5.390 & 0.781 & $\mathrm{H}$ & 8.349 & 5.295 & 0.807 \\
\hline $\mathrm{H}$ & 9.636 & 5.605 & -0.062 & $\mathrm{H}$ & 9.869 & 5.582 & -0.044 \\
\hline $\mathrm{C}$ & 15.028 & -5.364 & 1.104 & $\mathrm{C}$ & 15.800 & -5.110 & 1.082 \\
\hline $\mathrm{H}$ & 14.097 & -4.799 & 1.190 & $\mathrm{H}$ & 14.840 & -4.598 & 1.175 \\
\hline $\mathrm{H}$ & 14.777 & -6.289 & 0.568 & $\mathrm{H}$ & 15.596 & -6.048 & 0.547 \\
\hline $\mathrm{C}$ & 15.531 & -5.719 & 2.515 & $\mathrm{C}$ & 16.332 & -5.436 & 2.488 \\
\hline $\mathrm{H}$ & 16.446 & -6.319 & 2.478 & $\mathrm{H}$ & 17.279 & -5.984 & 2.445 \\
\hline $\mathrm{H}$ & 15.749 & -4.815 & 3.090 & $\mathrm{H}$ & 16.504 & -4.521 & 3.062 \\
\hline $\mathrm{H}$ & 14.775 & -6.295 & 3.058 & $\mathrm{H}$ & 15.613 & -6.053 & 3.037 \\
\hline $\mathrm{C}$ & -3.694 & 4.603 & 0.061 & $\mathrm{C}$ & -3.297 & 4.404 & 0.108 \\
\hline $\mathrm{H}$ & -4.390 & 4.452 & -0.773 & $\mathrm{H}$ & -3.990 & 4.282 & -0.733 \\
\hline $\mathrm{H}$ & -4.298 & 4.484 & 0.971 & $\mathrm{H}$ & -3.911 & 4.287 & 1.011 \\
\hline $\mathrm{C}$ & -3.163 & 6.041 & 0.009 & $\mathrm{C}$ & -2.732 & 5.828 & 0.081 \\
\hline $\mathrm{H}$ & -2.521 & 6.265 & 0.866 & $\mathrm{H}$ & -2.091 & 6.026 & 0.946 \\
\hline $\mathrm{H}$ & -2.586 & 6.224 & -0.901 & $\mathrm{H}$ & -2.144 & 6.011 & -0.823 \\
\hline $\mathrm{H}$ & -3.998 & 6.747 & 0.025 & $\mathrm{H}$ & -3.549 & 6.555 & 0.100 \\
\hline $\mathrm{C}$ & -10.867 & -3.398 & 0.178 & $\mathrm{C}$ & -10.662 & -3.411 & 0.093 \\
\hline $\mathrm{H}$ & -10.259 & -3.359 & -0.735 & $\mathrm{H}$ & -10.045 & -3.355 & -0.813 \\
\hline $\mathrm{H}$ & -10.174 & -3.171 & 0.999 & $\mathrm{H}$ & -9.973 & -3.230 & 0.927 \\
\hline $\mathrm{C}$ & -11.395 & -4.825 & 0.356 & $\mathrm{C}$ & -11.226 & -4.831 & 0.215 \\
\hline $\mathrm{H}$ & -11.981 & -4.925 & 1.275 & $\mathrm{H}$ & -11.820 & -4.951 & 1.126 \\
\hline $\mathrm{H}$ & -12.028 & -5.130 & -0.482 & $\mathrm{H}$ & -11.861 & -5.089 & -0.638 \\
\hline
\end{tabular}




\begin{tabular}{|c|c|c|c|c|c|c|c|}
\hline $\mathrm{H}$ & -10.559 & -5.528 & 0.414 & $\mathrm{H}$ & -10.408 & -5.556 & 0.250 \\
\hline $\mathrm{H}$ & -18.139 & 0.880 & 0.020 & $\mathrm{~F}$ & -8.210 & 2.767 & 0.410 \\
\hline $\mathrm{H}$ & -15.714 & 0.706 & 0.101 & $\mathrm{~F}$ & -5.762 & -1.766 & -0.341 \\
\hline $\mathrm{H}$ & 1.137 & 0.484 & -0.234 & $\mathrm{~F}$ & 10.859 & -2.935 & -0.693 \\
\hline $\mathrm{H}$ & 3.522 & 0.287 & -0.253 & $\mathrm{~F}$ & 13.915 & 1.128 & 0.424 \\
\hline $\mathrm{F}$ & -8.567 & 2.841 & 0.416 & $\mathrm{~F}$ & -17.988 & 1.153 & -0.111 \\
\hline $\mathrm{F}$ & -6.008 & -1.627 & -0.357 & $\mathrm{~F}$ & -15.414 & 1.005 & -0.043 \\
\hline $\mathrm{F}$ & 10.224 & -2.949 & -0.729 & $\mathrm{~F}$ & 1.444 & -0.022 & -0.097 \\
\hline $\mathrm{F}$ & 13.469 & 0.958 & 0.418 & $\mathrm{~F}$ & 3.972 & -0.203 & -0.119 \\
\hline \multicolumn{4}{|c|}{ BT4Tffo } & \multicolumn{4}{|c|}{ BTff4Tffo } \\
\hline $\mathrm{C}$ & 17.929 & -2.413 & -0.113 & $\mathrm{C}$ & 17.674 & -2.388 & -0.148 \\
\hline $\mathrm{C}$ & 16.478 & -2.530 & -0.150 & $\mathrm{C}$ & 16.219 & -2.488 & -0.152 \\
\hline $\mathrm{C}$ & 15.642 & -1.365 & 0.043 & $\mathrm{C}$ & 15.380 & -1.307 & -0.038 \\
\hline $\mathrm{C}$ & 16.321 & -0.175 & 0.260 & $\mathrm{C}$ & 16.096 & -0.128 & 0.068 \\
\hline $\mathrm{C}$ & 17.740 & -0.071 & 0.295 & $\mathrm{C}$ & 17.520 & -0.041 & 0.072 \\
\hline $\mathrm{C}$ & 18.556 & -1.157 & 0.114 & $\mathrm{C}$ & 18.329 & -1.132 & -0.032 \\
\hline $\mathrm{H}$ & 19.637 & -1.090 & 0.139 & $\mathrm{H}$ & 19.408 & -1.047 & -0.028 \\
\hline $\mathrm{C}$ & 14.187 & -1.434 & 0.011 & $\mathrm{C}$ & 13.926 & -1.364 & -0.038 \\
\hline $\mathrm{C}$ & 13.366 & -2.533 & -0.144 & $\mathrm{C}$ & 13.127 & -2.489 & -0.141 \\
\hline S & 13.217 & 0.014 & 0.189 & $\mathrm{~S}$ & 12.921 & 0.068 & 0.101 \\
\hline $\mathrm{C}$ & 11.976 & -2.254 & -0.124 & $\mathrm{C}$ & 11.734 & -2.242 & -0.113 \\
\hline $\mathrm{H}$ & 13.774 & -3.525 & -0.271 & $\mathrm{H}$ & 13.552 & -3.476 & -0.235 \\
\hline $\mathrm{C}$ & 11.714 & -0.893 & 0.051 & $\mathrm{C}$ & 11.445 & -0.881 & 0.016 \\
\hline $\mathrm{C}$ & 10.458 & -0.188 & 0.125 & $\mathrm{C}$ & 10.172 & -0.205 & 0.083 \\
\hline $\mathrm{C}$ & 10.244 & 1.167 & 0.311 & $\mathrm{C}$ & 9.926 & 1.149 & 0.229 \\
\hline S & 8.876 & -0.958 & -0.019 & $\mathrm{~S}$ & 8.608 & -1.019 & -0.019 \\
\hline $\mathrm{C}$ & 8.908 & 1.600 & 0.351 & $\mathrm{C}$ & 8.580 & 1.550 & 0.269 \\
\hline $\mathrm{C}$ & 8.017 & 0.558 & 0.190 & $\mathrm{C}$ & 7.714 & 0.482 & 0.150 \\
\hline $\mathrm{H}$ & 8.630 & 2.635 & 0.510 & $\mathrm{H}$ & 8.278 & 2.582 & 0.396 \\
\hline $\mathrm{C}$ & 6.578 & 0.592 & 0.173 & $\mathrm{C}$ & 6.274 & 0.480 & 0.147 \\
\hline $\mathrm{C}$ & 5.685 & -0.459 & 0.200 & $\mathrm{C}$ & 5.409 & -0.594 & 0.186 \\
\hline S & 5.722 & 2.124 & 0.104 & $\mathrm{~S}$ & 5.379 & 1.989 & 0.083 \\
\hline $\mathrm{C}$ & 4.349 & -0.024 & 0.171 & $\mathrm{C}$ & 4.062 & -0.193 & 0.168 \\
\hline $\mathrm{H}$ & 5.961 & -1.505 & 0.253 & $\mathrm{H}$ & 5.711 & -1.632 & 0.239 \\
\hline $\mathrm{C}$ & 4.137 & 1.344 & 0.126 & $\mathrm{C}$ & 3.815 & 1.169 & 0.120 \\
\hline $\mathrm{C}$ & 2.883 & 2.053 & 0.095 & $\mathrm{C}$ & 2.542 & 1.845 & 0.096 \\
\hline $\mathrm{C}$ & 2.620 & 3.425 & 0.060 & $\mathrm{C}$ & 2.249 & 3.212 & 0.068 \\
\hline S & 1.378 & 1.135 & 0.095 & $\mathrm{~S}$ & 1.067 & 0.888 & 0.094 \\
\hline $\mathrm{C}$ & 1.232 & 3.705 & 0.034 & $\mathrm{C}$ & 0.857 & 3.458 & 0.047 \\
\hline $\mathrm{C}$ & 0.409 & 2.595 & 0.047 & $\mathrm{C}$ & 0.059 & 2.326 & 0.056 \\
\hline $\mathrm{H}$ & 0.826 & 4.705 & 0.007 & $\mathrm{H}$ & 0.431 & 4.448 & 0.026 \\
\hline $\mathrm{N}$ & 18.568 & -3.573 & -0.309 & $\mathrm{~N}$ & 18.296 & -3.565 & -0.261 \\
\hline $\mathrm{N}$ & 16.061 & -3.781 & -0.376 & $\mathrm{~N}$ & 15.788 & -3.745 & -0.269 \\
\hline S & 17.407 & -4.714 & -0.524 & $\mathrm{~S}$ & 17.120 & -4.708 & -0.364 \\
\hline
\end{tabular}




\begin{tabular}{|c|c|c|c|c|c|c|c|}
\hline $\mathrm{C}$ & -1.041 & 2.520 & 0.026 & $\mathrm{~F}$ & 18.033 & 1.190 & 0.185 \\
\hline $\mathrm{C}$ & -1.888 & 3.689 & -0.003 & $\mathrm{~F}$ & 15.458 & 1.050 & 0.181 \\
\hline $\mathrm{C}$ & -1.725 & 1.310 & 0.031 & $\mathrm{C}$ & -1.391 & 2.261 & 0.037 \\
\hline $\mathrm{C}$ & -3.345 & 3.573 & -0.026 & $\mathrm{C}$ & -2.246 & 3.430 & 0.011 \\
\hline $\mathrm{N}$ & -1.465 & 4.956 & -0.013 & $\mathrm{C}$ & -2.108 & 1.078 & 0.039 \\
\hline $\mathrm{C}$ & -3.130 & 1.199 & 0.009 & $\mathrm{C}$ & -3.707 & 3.329 & -0.009 \\
\hline $\mathrm{C}$ & -3.997 & 2.284 & -0.020 & $\mathrm{~N}$ & -1.813 & 4.691 & 0.005 \\
\hline $\mathrm{N}$ & -3.962 & 4.758 & -0.053 & $\mathrm{C}$ & -3.521 & 0.981 & 0.018 \\
\hline S & -2.797 & 5.915 & -0.049 & $\mathrm{~F}$ & -1.467 & -0.103 & 0.062 \\
\hline $\mathrm{C}$ & -5.440 & 2.129 & -0.043 & $\mathrm{C}$ & -4.394 & 2.054 & -0.007 \\
\hline $\mathrm{C}$ & -6.430 & 3.094 & -0.065 & $\mathrm{~N}$ & -4.308 & 4.519 & -0.031 \\
\hline S & -6.165 & 0.532 & -0.046 & $\mathrm{~S}$ & -3.134 & 5.666 & -0.025 \\
\hline $\mathrm{C}$ & -7.756 & 2.595 & -0.082 & $\mathrm{~F}$ & -3.994 & -0.277 & 0.024 \\
\hline $\mathrm{H}$ & -6.189 & 4.146 & -0.067 & $\mathrm{C}$ & -5.839 & 1.917 & -0.030 \\
\hline $\mathrm{C}$ & -7.796 & 1.198 & -0.075 & $\mathrm{C}$ & -6.786 & 2.929 & -0.051 \\
\hline $\mathrm{C}$ & -8.922 & 0.298 & -0.088 & $\mathrm{~S}$ & -6.641 & 0.354 & -0.036 \\
\hline $\mathrm{C}$ & -8.913 & -1.086 & -0.077 & $\mathrm{C}$ & -8.131 & 2.493 & -0.072 \\
\hline S & -10.610 & 0.816 & -0.118 & $\mathrm{H}$ & -6.499 & 3.968 & -0.052 \\
\hline $\mathrm{C}$ & -10.163 & -1.730 & -0.106 & $\mathrm{C}$ & -8.233 & 1.099 & -0.067 \\
\hline $\mathrm{C}$ & -11.211 & -0.833 & -0.137 & $\mathrm{C}$ & -9.401 & 0.253 & -0.082 \\
\hline $\mathrm{H}$ & -10.269 & -2.807 & -0.123 & $\mathrm{C}$ & -9.459 & -1.130 & -0.083 \\
\hline $\mathrm{C}$ & -12.627 & -1.096 & -0.176 & $\mathrm{~S}$ & -11.063 & 0.852 & -0.100 \\
\hline $\mathrm{C}$ & -13.663 & -0.227 & -0.447 & $\mathrm{C}$ & -10.738 & -1.712 & -0.111 \\
\hline $\mathrm{S}$ & -13.249 & -2.703 & 0.167 & $\mathrm{C}$ & -11.742 & -0.766 & -0.128 \\
\hline $\mathrm{C}$ & -14.920 & -0.858 & -0.373 & $\mathrm{H}$ & -10.896 & -2.784 & -0.135 \\
\hline $\mathrm{H}$ & -13.544 & 0.818 & -0.701 & $\mathrm{C}$ & -13.169 & -0.961 & -0.161 \\
\hline $\mathrm{C}$ & -14.924 & -2.201 & -0.051 & $\mathrm{C}$ & -14.166 & -0.038 & -0.400 \\
\hline $\mathrm{C}$ & -16.057 & -3.099 & 0.049 & $\mathrm{~S}$ & -13.863 & -2.546 & 0.146 \\
\hline $\mathrm{C}$ & -16.185 & -4.331 & 0.680 & $\mathrm{C}$ & -15.451 & -0.612 & -0.331 \\
\hline S & -17.571 & -2.649 & -0.741 & $\mathrm{H}$ & -14.000 & 1.006 & -0.627 \\
\hline $\mathrm{C}$ & -17.489 & -4.889 & 0.508 & $\mathrm{C}$ & -15.514 & -1.962 & -0.044 \\
\hline $\mathrm{C}$ & -18.335 & -4.110 & -0.227 & $\mathrm{C}$ & -16.689 & -2.807 & 0.046 \\
\hline $\mathrm{H}$ & -17.779 & -5.840 & 0.940 & $\mathrm{C}$ & -16.862 & -4.059 & 0.624 \\
\hline $\mathrm{H}$ & -19.369 & -4.296 & -0.479 & $\mathrm{~S}$ & -18.197 & -2.246 & -0.682 \\
\hline $\mathrm{C}$ & -8.975 & 3.492 & -0.105 & $\mathrm{C}$ & -18.198 & -4.543 & 0.462 \\
\hline $\mathrm{H}$ & -9.582 & 3.253 & -0.989 & $\mathrm{C}$ & -19.020 & -3.690 & -0.214 \\
\hline $\mathrm{H}$ & -9.611 & 3.260 & 0.759 & $\mathrm{H}$ & -18.524 & -5.499 & 0.858 \\
\hline $\mathrm{C}$ & -8.681 & 4.996 & -0.106 & $\mathrm{H}$ & -20.069 & -3.814 & -0.447 \\
\hline $\mathrm{H}$ & -8.127 & 5.299 & 0.787 & $\mathrm{C}$ & -9.306 & 3.447 & -0.096 \\
\hline $\mathrm{H}$ & -8.097 & 5.292 & -0.983 & $\mathrm{H}$ & -9.926 & 3.234 & -0.978 \\
\hline $\mathrm{H}$ & -9.618 & 5.560 & -0.124 & $\mathrm{H}$ & -9.950 & 3.249 & 0.770 \\
\hline $\mathrm{C}$ & -15.127 & -5.060 & 1.475 & $\mathrm{C}$ & -8.941 & 4.936 & -0.104 \\
\hline $\mathrm{H}$ & -14.412 & -4.351 & 1.903 & $\mathrm{H}$ & -8.373 & 5.216 & 0.788 \\
\hline $\mathrm{H}$ & -15.617 & -5.544 & 2.328 & $\mathrm{H}$ & -8.343 & 5.200 & -0.981 \\
\hline
\end{tabular}




\begin{tabular}{rrrrrrrr} 
C & -14.374 & -6.130 & 0.662 & $\mathrm{H}$ & -9.850 & 5.544 & -0.125 \\
$\mathrm{H}$ & -15.067 & -6.878 & 0.264 & $\mathrm{C}$ & -15.824 & -4.880 & 1.352 \\
$\mathrm{H}$ & -13.844 & -5.687 & -0.185 & $\mathrm{H}$ & -15.064 & -4.231 & 1.798 \\
$\mathrm{H}$ & -13.641 & -6.647 & 1.289 & $\mathrm{H}$ & -16.318 & -5.384 & 2.191 \\
$\mathrm{C}$ & 3.680 & 4.506 & 0.049 & $\mathrm{C}$ & -15.147 & -5.942 & 0.465 \\
$\mathrm{H}$ & 4.325 & 4.388 & 0.930 & $\mathrm{H}$ & -15.887 & -6.631 & 0.047 \\
$\mathrm{H}$ & 4.337 & 4.361 & -0.818 & $\mathrm{H}$ & -14.614 & -5.482 & -0.372 \\
$\mathrm{C}$ & 3.146 & 5.943 & 0.023 & $\mathrm{H}$ & -14.427 & -6.528 & 1.045 \\
$\mathrm{H}$ & 2.544 & 6.132 & -0.869 & $\mathrm{C}$ & 3.282 & 4.320 & 0.062 \\
$\mathrm{H}$ & 2.528 & 6.159 & 0.900 & $\mathrm{H}$ & 3.936 & 4.208 & 0.937 \\
$\mathrm{H}$ & 3.980 & 6.652 & 0.020 & $\mathrm{H}$ & 3.935 & 4.200 & -0.812 \\
$\mathrm{C}$ & 10.917 & -3.324 & -0.279 & $\mathrm{C}$ & 2.713 & 5.743 & 0.055 \\
$\mathrm{H}$ & 10.249 & -3.300 & 0.592 & $\mathrm{H}$ & 2.101 & 5.928 & -0.832 \\
$\mathrm{H}$ & 10.283 & -3.083 & -1.143 & $\mathrm{H}$ & 2.095 & 5.934 & 0.937 \\
$\mathrm{C}$ & 11.450 & -4.751 & -0.448 & $\mathrm{H}$ & 3.529 & 6.472 & 0.055 \\
$\mathrm{H}$ & 12.075 & -4.846 & -1.342 & $\mathrm{C}$ & 10.700 & -3.344 & -0.213 \\
$\mathrm{H}$ & 12.046 & -5.064 & 0.414 & $\mathrm{H}$ & 10.042 & -3.301 & 0.664 \\
$\mathrm{H}$ & 10.617 & -5.452 & -0.548 & $\mathrm{H}$ & 10.051 & -3.152 & -1.078 \\
$\mathrm{H}$ & 18.176 & 0.906 & 0.472 & $\mathrm{C}$ & 11.266 & -4.763 & -0.331 \\
$\mathrm{H}$ & 15.752 & 0.735 & 0.417 & $\mathrm{H}$ & 11.880 & -4.879 & -1.229 \\
$\mathrm{H}$ & -1.159 & 0.384 & 0.053 & $\mathrm{H}$ & 11.881 & -5.025 & 0.534 \\
$\mathrm{H}$ & -3.543 & 0.195 & 0.017 & $\mathrm{H}$ & 10.449 & -5.487 & -0.391 \\
$\mathrm{~F}$ & 11.260 & 2.040 & 0.456 & $\mathrm{~F}$ & 10.920 & 2.050 & 0.334 \\
$\mathrm{~F}$ & 3.333 & -0.906 & 0.196 & $\mathrm{~F}$ & 3.069 & -1.100 & 0.204 \\
$\mathrm{~F}$ & -7.769 & -1.796 & -0.052 & $\mathrm{~F}$ & -8.351 & -1.893 & -0.070 \\
$\mathrm{~F}$ & -16.053 & -0.176 & -0.626 & $\mathrm{~F}$ & -16.552 & 0.128 & -0.555 \\
\hline & & & & & & &
\end{tabular}
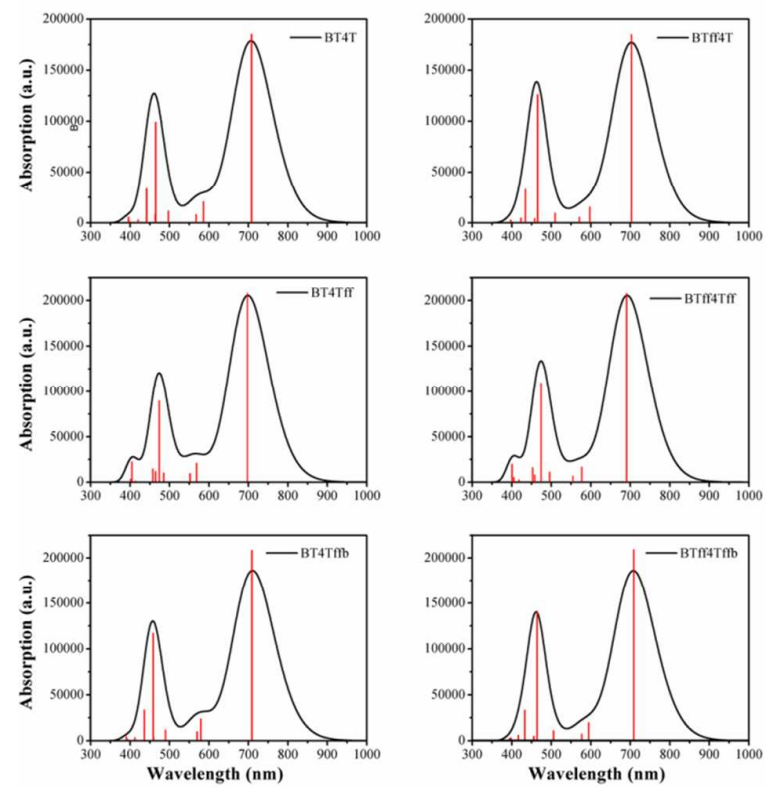
Figure S2. Calculated absorption spectra of BT4T and its fluorinated derivatives.

Table S2. Calculated electronic transitions energy $E(\mathrm{eV})$, absorption wavelength $\lambda(\mathrm{nm})$, transition electronic dipole moments $\mu_{\mathrm{tr}}(\mathrm{D})$, oscillator strength $(f)$, and main configurations of BT4T and its derivatives

\begin{tabular}{|c|c|c|c|c|c|c|c|}
\hline & states & $E(\mathrm{eV})$ & $\lambda(\mathrm{nm})$ & $\mu_{\mathrm{tr}}(\mathrm{D})$ & $f$ & main config. & $\exp . \lambda(\mathrm{nm})$ \\
\hline BT4T & $\begin{array}{l}\mathrm{S}_{0} \rightarrow \mathrm{S}_{1} \\
\mathrm{~S}_{0} \rightarrow \mathrm{S}_{2} \\
\mathrm{~S}_{0} \rightarrow \mathrm{S}_{4} \\
\mathrm{~S}_{0} \rightarrow \mathrm{S}_{6} \\
\mathrm{~S}_{0} \rightarrow \mathrm{S}_{7} \\
\mathrm{~S}_{0} \rightarrow \mathrm{S}_{10}\end{array}$ & $\begin{array}{l}1.659 \\
1.954 \\
2.297 \\
2.533 \\
2.695 \\
2.949\end{array}$ & $\begin{array}{l}747.2 \\
634.5 \\
539.8 \\
489.4 \\
460.1 \\
420.4\end{array}$ & $\begin{array}{l}44.953 \\
2.542 \\
2.499 \\
18.991 \\
4.196 \\
1.585\end{array}$ & $\begin{array}{l}1.827 \\
0.122 \\
0.1406 \\
1.179 \\
0.277 \\
0.115\end{array}$ & $\begin{array}{l}\mathrm{H} \rightarrow \mathrm{L}(67 \%) \\
\mathrm{H} \rightarrow \mathrm{L}+1(69 \%) \\
\mathrm{H}-1 \rightarrow \mathrm{L}+1(68 \%) \\
\mathrm{H} \rightarrow \mathrm{L}+2(61 \%) \\
\mathrm{H} \rightarrow \mathrm{L}+3(61 \%) \\
\mathrm{H}-1 \rightarrow \mathrm{L}+2(64 \%)\end{array}$ & 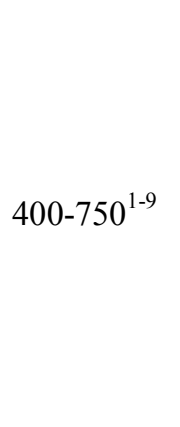 \\
\hline BTff4T & $\begin{array}{l}\mathrm{S}_{0} \rightarrow \mathrm{S}_{1} \\
\mathrm{~S}_{0} \rightarrow \mathrm{S}_{4} \\
\mathrm{~S}_{0} \rightarrow \mathrm{S}_{5} \\
\mathrm{~S}_{0} \rightarrow \mathrm{S}_{8}\end{array}$ & $\begin{array}{l}1.650 \\
2.218 \\
2.519 \\
2.736\end{array}$ & $\begin{array}{l}751.3 \\
559.1 \\
492.1 \\
453.1\end{array}$ & $\begin{array}{l}43.349 \\
2.088 \\
22.880 \\
3.490\end{array}$ & $\begin{array}{l}1.753 \\
0.114 \\
1.412 \\
0.234\end{array}$ & $\begin{array}{l}H \rightarrow L(70 \%) \\
H-1 \rightarrow L+1(68 \%) \\
H \rightarrow L+2(65 \%) \\
H \rightarrow L+3(57 \%)\end{array}$ & \\
\hline BT4Tffi & $\begin{array}{l}\mathrm{S}_{0} \rightarrow \mathrm{S}_{1} \\
\mathrm{~S}_{0} \rightarrow \mathrm{S}_{2} \\
\mathrm{~S}_{0} \rightarrow \mathrm{S}_{3} \\
\mathrm{~S}_{0} \rightarrow \mathrm{S}_{4} \\
\mathrm{~S}_{0} \rightarrow \mathrm{S}_{5} \\
\mathrm{~S}_{0} \rightarrow \mathrm{S}_{6} \\
\mathrm{~S}_{0} \rightarrow \mathrm{S}_{7} \\
\mathrm{~S}_{0} \rightarrow \mathrm{S}_{10}\end{array}$ & $\begin{array}{l}1.644 \\
1.959 \\
2.021 \\
2.306 \\
2.525 \\
2.542 \\
2.702 \\
2.958\end{array}$ & $\begin{array}{l}754.0 \\
632.9 \\
613.3 \\
537.8 \\
491.1 \\
487.7 \\
458.9 \\
419.2\end{array}$ & $\begin{array}{l}47.174 \\
2.807 \\
2.049 \\
2.302 \\
3.118 \\
17.617 \\
3.891 \\
1.396\end{array}$ & $\begin{array}{l}1.900 \\
0.135 \\
0.101 \\
0.130 \\
0.193 \\
1.097 \\
0.258 \\
0.101\end{array}$ & $\begin{array}{l}H \rightarrow L(70 \%) \\
H \rightarrow L+1(67 \%) \\
H-1 \rightarrow L(67 \%) \\
H-1 \rightarrow L+1(68 \%) \\
H-2 \rightarrow L(65 \%) \\
H \rightarrow L+2(59 \%) \\
H \rightarrow L+3(61 \%) \\
H-1 \rightarrow L+2(56 \%)\end{array}$ & \\
\hline BTff4Tffi & $\begin{array}{l}\mathrm{S}_{0} \rightarrow \mathrm{S}_{1} \\
\mathrm{~S}_{0} \rightarrow \mathrm{S}_{2} \\
\mathrm{~S}_{0} \rightarrow \mathrm{S}_{4} \\
\mathrm{~S}_{0} \rightarrow \mathrm{S}_{5} \\
\mathrm{~S}_{0} \rightarrow \mathrm{S}_{7}\end{array}$ & $\begin{array}{l}1.632 \\
1.904 \\
2.223 \\
2.516 \\
2.728\end{array}$ & $\begin{array}{l}759.8 \\
651.3 \\
557.7 \\
492.7 \\
454.5\end{array}$ & $\begin{array}{l}46.041 \\
2.355 \\
1.948 \\
24.440 \\
3.330\end{array}$ & $\begin{array}{l}1.841 \\
0.110 \\
0.106 \\
1.507 \\
0.223\end{array}$ & $\begin{array}{l}H \rightarrow L(70 \%) \\
H \rightarrow L+1(69 \%) \\
H-1 \rightarrow L+1(69 \%) \\
H \rightarrow L+2(66 \%) \\
H \rightarrow L+3(65 \%)\end{array}$ & \\
\hline
\end{tabular}




\begin{tabular}{|c|c|c|c|c|c|c|}
\hline \multirow{9}{*}{ BT4Tffo } & $\mathrm{S}_{0} \rightarrow \mathrm{S}_{1}$ & 1.679 & 738.2 & 51.578 & 2.122 & $\mathrm{H} \rightarrow \mathrm{L}(70 \%)$ \\
\hline & $\mathrm{S}_{0} \rightarrow \mathrm{S}_{2}$ & 2.001 & 619.7 & 2.068 & 0.101 & $\mathrm{H} \rightarrow \mathrm{L}+1(68 \%)$ \\
\hline & $\mathrm{S}_{0} \rightarrow \mathrm{S}_{3}$ & 2.091 & 592.9 & 2.314 & 0.119 & $\mathrm{H}-1 \rightarrow \mathrm{L}(67 \%)$ \\
\hline & $\mathrm{S}_{0} \rightarrow \mathrm{S}_{4}$ & 2.349 & 527.8 & 2.982 & 0.172 & $\mathrm{H}-1 \rightarrow \mathrm{L}+1(68 \%)$ \\
\hline & $\mathrm{S}_{0} \rightarrow \mathrm{S}_{5}$ & 2.456 & 504.9 & 15.218 & 0.916 & $\mathrm{H} \rightarrow \mathrm{L}+3(65 \%)$ \\
\hline & $\mathrm{S}_{0} \rightarrow \mathrm{S}_{6}$ & 2.498 & 496.3 & 1.846 & 0.113 & $\mathrm{H}-2 \rightarrow \mathrm{L}(59 \%)$ \\
\hline & $\mathrm{S}_{0} \rightarrow \mathrm{S}_{7}$ & 2.567 & 483.0 & 3.404 & 0.214 & $\mathrm{H} \rightarrow \mathrm{L}+2(61 \%)$ \\
\hline & $\mathrm{S}_{0} \rightarrow \mathrm{S}_{9}$ & 2.874 & 431.5 & 3.582 & 0.252 & $\mathrm{H}-1 \rightarrow \mathrm{L}+2(52 \%)$ \\
\hline & $\mathrm{S}_{0} \rightarrow \mathrm{S}_{10}$ & 2.894 & 428.5 & 2.195 & 0.156 & $\mathrm{H}-1 \rightarrow \mathrm{L}+3(59 \%)$ \\
\hline \multirow{5}{*}{ BTff4Tffo } & $\mathrm{S}_{0} \rightarrow \mathrm{S}_{1}$ & 1.679 & 738.5 & 49.893 & 2.052 & $\mathrm{H} \rightarrow \mathrm{L}(70 \%)$ \\
\hline & $\mathrm{S}_{0} \rightarrow \mathrm{S}_{4}$ & 2.280 & 543.9 & 2.587 & 0.145 & $\mathrm{H}-1 \rightarrow \mathrm{L}+1(68 \%)$ \\
\hline & $\mathrm{S}_{0} \rightarrow \mathrm{S}_{5}$ & 2.453 & 505.5 & 21.035 & 1.264 & $\mathrm{H} \rightarrow \mathrm{L}+2(53 \%)$ \\
\hline & $\mathrm{S}_{0} \rightarrow \mathrm{S}_{7}$ & 2.593 & 478.1 & 3.441 & 0.219 & $\mathrm{H} \rightarrow \mathrm{L}+3(53 \%)$ \\
\hline & $\mathrm{S}_{0} \rightarrow \mathrm{S}_{9}$ & 2.848 & 435.4 & 1.715 & 0.120 & $\mathrm{H}-1 \rightarrow \mathrm{L}+2(63 \%)$ \\
\hline
\end{tabular}

The influence of functionals, basis sets and structure relaxation on the total energies, HOMO, LUMO energies and band gaps of polymers.

The total energies, FMOs and band gaps are calculated based on various methods, such as B3LYP, O3LYP, PBE0 and cam-B3LYP, all of which are widely used in predicting photoelectric properties in organo semiconductors, listed in Table S3. By comparing the results, one can observe that the variation trend of HOMOs is consistent, i.e. the HOMO values of F-substituted polymers decrease with increasing F number per repeating unit, and that the electronic band gaps basically remain unchanged with various $\mathrm{F}$ substitutions, only outer position fluorination increase the band gaps a little, leading to a slightly blue-shifted absorption spectra. As an example, the HOMO energies and band gaps of polymers are calculated with different exchange and correlation functionals and long range corrections under 6-311G(d,p) basis set, and plotted in Figure S3. The energy dependences on substituted F atoms show almost the same trend for different functionals, thus proving the credible results. 
Furthermore, the influence of the basis set as well as the structure relaxation is also investigated. As can be seen in Table S4, larger basis sets give more stable structures and lower total energies, however, the deviation of the polymer backbone from planarity is also occurred, due to the introduction of polarization functions, which lead to a wider band gap. It is found that based on structure optimization with $6-31 \mathrm{G}$ basis set, followed by more accurate single-point energy calculation with more flexible 6-311G(d,p) basis set, rationable values for both HOMO and band gap energies could be obtained, which are in agreement with the experimental results. This strategy was often used in studies on similar organo semiconductor systems. ${ }^{10}$

Table S3. Total energies, HOMO, LUMO energies and band gaps of polymers with various functionals and basis sets. The geometries are optimized under 6-31G basis set. (Unit: total energy in Hartree, HOMO, LUMO energies and band gap in eV)

\begin{tabular}{|c|c|c|c|c|c|c|}
\hline & BT4T & BTff4T & BT4Tffi & BTff4Tffi & BT4Tffo & BTff4Tffo \\
\hline \multicolumn{7}{|c|}{ B3LYP/6-31G } \\
\hline Energy & -6204.34 & -6601.16 & -6601.17 & -6997.99 & -6601.16 & -6997.99 \\
\hline HOMO & -4.78 & -4.85 & -4.96 & -5.08 & -4.95 & -5.08 \\
\hline LUMO & -3.15 & -3.34 & -3.29 & -3.48 & -3.23 & -3.43 \\
\hline $\mathrm{Eg}$ & 1.63 & 1.51 & 1.67 & 1.59 & 1.71 & 1.65 \\
\hline \multicolumn{7}{|c|}{ B3LYP/6-31G(d) } \\
\hline Energy & -6205.22 & -6602.13 & -6602.13 & -6999.04 & -6602.13 & -6999.04 \\
\hline HOMO & -4.69 & -4.74 & -4.82 & -4.90 & -4.81 & -4.91 \\
\hline LUMO & -3.05 & -3.17 & -3.16 & -3.29 & -3.11 & -3.24 \\
\hline $\mathrm{Eg}$ & 1.63 & 1.56 & 1.65 & 1.61 & 1.70 & 1.67 \\
\hline \multicolumn{7}{|c|}{ B3LYP/6-311G(d,p) } \\
\hline Energy & -6205.97 & -6603.00 & -6603.01 & -7000.04 & -6603.00 & -7000.03 \\
\hline HOMO & -4.91 & -4.97 & -5.06 & -5.16 & -5.05 & -5.16 \\
\hline LUMO & -3.29 & -3.42 & -3.41 & -3.54 & -3.36 & -3.49 \\
\hline $\mathrm{Eg}$ & 1.61 & 1.55 & 1.64 & 1.61 & 1.69 & 1.67 \\
\hline
\end{tabular}




\begin{tabular}{|c|c|c|c|c|c|c|}
\hline \multicolumn{7}{|c|}{ O3LYP/6-31G } \\
\hline Energy & -6203.41 & -6600.12 & -6601.17 & -6996.82 & -6600.12 & -6996.82 \\
\hline HOMO & -4.50 & -4.62 & -4.96 & -4.79 & -4.68 & -4.81 \\
\hline LUMO & -3.23 & -3.42 & -3.29 & -3.55 & -3.32 & -3.50 \\
\hline $\mathrm{Eg}$ & 1.27 & 1.20 & 1.67 & 1.24 & 1.35 & 1.31 \\
\hline \multicolumn{7}{|c|}{ O3LYP/6-31G(d) } \\
\hline Energy & -6204.25 & -6601.05 & -6601.04 & -6997.84 & -6601.05 & -6997.84 \\
\hline HOMO & -4.42 & -4.51 & -4.55 & -4.62 & -4.55 & -4.65 \\
\hline LUMO & -3.15 & -3.26 & -3.26 & -3.36 & -3.21 & -3.32 \\
\hline $\mathrm{Eg}$ & 1.27 & 1.24 & 1.29 & 1.26 & 1.33 & 1.32 \\
\hline \multicolumn{7}{|c|}{ O3LYP/6-311G(d,p) } \\
\hline Energy & -6204.96 & -6601.87 & -6601.86 & -6998.78 & -6601.87 & -6998.77 \\
\hline HOMO & -4.59 & -4.69 & -4.73 & -4.82 & -4.73 & -4.84 \\
\hline LUMO & -3.33 & -3.45 & -3.45 & -3.56 & -3.40 & -3.52 \\
\hline $\mathrm{Eg}$ & 1.25 & 1.23 & 1.28 & 1.26 & 1.32 & 1.32 \\
\hline \multicolumn{7}{|c|}{ PBE1PBE /6-31G } \\
\hline Energy & -6200.25 & -6596.72 & -6596.73 & -6993.19 & -6596.72 & -6993.19 \\
\hline HOMO & -5.00 & -5.07 & -5.14 & -5.29 & -5.17 & -5.29 \\
\hline LUMO & -3.09 & -3.27 & -3.21 & -3.42 & -3.16 & -3.35 \\
\hline $\mathrm{Eg}$ & 1.91 & 1.79 & 1.92 & 1.87 & 2.00 & 1.94 \\
\hline \multicolumn{7}{|c|}{ PBE1PBE /6-31G(d) } \\
\hline Energy & -6201.15 & -6597.72 & -6597.72 & -6994.28 & -6597.71 & -6994.28 \\
\hline HOMO & -4.91 & -4.95 & -5.00 & -5.11 & -5.03 & -5.12 \\
\hline LUMO & -2.99 & -3.10 & -3.09 & -3.21 & -3.04 & -3.15 \\
\hline $\mathrm{Eg}$ & 1.91 & 1.85 & 1.90 & 1.90 & 1.98 & 1.96 \\
\hline \multicolumn{7}{|c|}{ PBE1PBE /6-311G(d,p) } \\
\hline Energy & -6201.84 & -6598.51 & -6598.52 & -6995.19 & -6598.51 & -6995.19 \\
\hline HOMO & -5.11 & -5.21 & -5.20 & -5.29 & -5.19 & -5.29 \\
\hline LUMO & -3.17 & -3.28 & -3.29 & -3.39 & -3.23 & -3.33 \\
\hline
\end{tabular}




\begin{tabular}{|c|c|c|c|c|c|c|}
\hline $\mathrm{Eg}$ & 1.94 & 1.93 & 1.91 & 1.90 & 1.96 & 1.96 \\
\hline \multicolumn{7}{|c|}{ Cam-B3LYP/6-31G } \\
\hline Energy & -6202.95 & -6599.70 & -6599.71 & -6996.46 & -6599.70 & -6996.45 \\
\hline HOMO & -6.01 & -6.08 & -6.19 & -6.30 & -6.19 & -6.31 \\
\hline LUMO & -2.11 & -2.34 & -2.25 & -2.48 & -2.18 & -2.40 \\
\hline $\mathrm{Eg}$ & 3.89 & 3.73 & 3.93 & 3.82 & 4.01 & 3.91 \\
\hline \multicolumn{7}{|c|}{ Cam-B3LYP/6-31G(d) } \\
\hline Energy & -6203.89 & -6600.73 & -6600.73 & -6997.57 & -6600.73 & -6997.57 \\
\hline HOMO & -5.91 & -5.95 & -6.03 & -6.11 & -6.03 & -6.12 \\
\hline LUMO & -2.00 & -2.14 & -2.11 & -2.25 & -2.04 & -2.17 \\
\hline Eg & 3.90 & 3.81 & 3.91 & 3.85 & 3.99 & 3.95 \\
\hline \multicolumn{7}{|c|}{ Cam-B3LYP/6-311G(d,p) } \\
\hline Energy & -6204.65 & -6601.61 & -6601.62 & -6998.58 & -6601.61 & -6998.58 \\
\hline HOMO & -6.12 & -6.18 & -6.26 & -6.36 & -6.27 & -6.37 \\
\hline LUMO & -2.25 & -2.39 & -2.37 & -2.51 & -2.30 & -2.44 \\
\hline $\mathrm{Eg}$ & 3.87 & 3.78 & 3.89 & 3.84 & 3.96 & 3.93 \\
\hline
\end{tabular}

Table S4. Total energies, HOMO, LUMO energies and band gaps of polymers with various functionals and basis sets. The geometries are optimized under 6-31G, 6-31G(d) and 6-311G(d,p) basis set, respectively. (Unit: total energy in Hartree, HOMO, LUMO energies and band gap in $\mathrm{eV})$

\begin{tabular}{l|c|c|c|c|c|c}
\hline & BT4T & BTff4T & BT4Tffi & BTff4Tffi & BT4Tffo & BTff4Tffo \\
\hline \multicolumn{5}{l}{ B3LYP/6-31G } \\
\hline Energy & -6204.34 & -6601.16 & -6601.17 & -6997.99 & -6601.16 & -6997.99 \\
HOMO & -4.78 & -4.85 & -4.96 & -5.08 & -4.95 & -5.08 \\
LUMO & -3.15 & -3.34 & -3.29 & -3.48 & -3.23 & -3.43 \\
\multicolumn{1}{c}{ Eg } & 1.63 & 1.51 & 1.67 & 1.59 & 1.71 & 1.65 \\
\hline
\end{tabular}

B3LYP/6-31G(d)

\begin{tabular}{l|l|l|l|l|l|l}
\hline Energy & -6205.27 & -6602.19 & -6602.19 & -6999.10 & -6602.19 & -6999.10
\end{tabular}




\begin{tabular}{|c|c|c|c|c|c|c|}
\hline HOMO & -4.70 & -4.78 & -4.77 & -4.85 & -4.77 & -4.85 \\
\hline LUMO & -2.75 & -2.84 & -2.85 & -2.94 & -2.80 & -2.89 \\
\hline $\mathrm{Eg}$ & 1.94 & 1.94 & 1.92 & 1.91 & 1.96 & 1.96 \\
\hline \multicolumn{7}{|c|}{ B3LYP/6-311G(d,p) } \\
\hline Energy & -6206.03 & -6603.07 & -6603.07 & -7000.10 & -6603.06 & -7000.10 \\
\hline HOMO & -5.16 & -5.14 & -5.12 & -5.20 & -5.04 & -5.14 \\
\hline LUMO & -2.93 & -3.04 & -3.04 & -3.14 & -3.03 & -3.12 \\
\hline $\mathrm{Eg}$ & 2.23 & 2.10 & 2.07 & 2.05 & 2.00 & 2.02 \\
\hline \multicolumn{7}{|c|}{ O3LYP/6-31G } \\
\hline Energy & -6203.41 & -6600.12 & -6601.17 & -6996.82 & -6600.12 & -6996.82 \\
\hline HOMO & -4.50 & -4.62 & -4.96 & -4.79 & -4.68 & -4.81 \\
\hline LUMO & -3.23 & -3.42 & -3.29 & -3.55 & -3.32 & -3.50 \\
\hline $\mathrm{Eg}$ & 1.27 & 1.20 & 1.67 & 1.24 & 1.35 & 1.31 \\
\hline \multicolumn{7}{|c|}{ O3LYP/6-31G(d) } \\
\hline Energy & -6204.31 & -6601.11 & -6601.11 & -6997.91 & -6601.11 & -6997.91 \\
\hline HOMO & -4.65 & -4.71 & -4.71 & -4.73 & -4.50 & -4.58 \\
\hline LUMO & -2.80 & -2.89 & -2.88 & -2.97 & -2.90 & -2.96 \\
\hline $\mathrm{Eg}$ & 1.85 & 1.81 & 1.82 & 1.75 & 1.59 & 1.61 \\
\hline \multicolumn{7}{|c|}{ O3LYP/6-311G(d,p) } \\
\hline Energy & -6205.02 & -6601.94 & -6601.94 & -6998.85 & -6601.94 & -6998.85 \\
\hline HOMO & -4.86 & -4.93 & -4.92 & -5.00 & -5.06 & -5.20 \\
\hline LUMO & -2.96 & -3.06 & -3.05 & -3.15 & -3.05 & -3.12 \\
\hline $\mathrm{Eg}$ & 1.89 & 1.86 & 1.87 & 1.84 & 2.01 & 2.08 \\
\hline
\end{tabular}

PBE1PBE /6-31G

\begin{tabular}{l|c|c|c|c|c|c}
\hline Energy & -6200.25 & -6596.72 & -6596.73 & -6993.19 & -6596.72 & -6993.19 \\
HOMO & -5.00 & -5.07 & -5.14 & -5.29 & -5.17 & -5.29 \\
LUMO & -3.09 & -3.27 & -3.21 & -3.42 & -3.16 & -3.35 \\
Eg & 1.91 & 1.79 & 1.92 & 1.87 & 2.00 & 1.94 \\
\hline
\end{tabular}




\begin{tabular}{|c|c|c|c|c|c|c|}
\hline Energy & -6201.21 & -6597.78 & -6597.78 & -6994.35 & -6597.78 & -6994.34 \\
\hline HOMO & -4.94 & -5.00 & -5.00 & -5.07 & -5.00 & -5.08 \\
\hline LUMO & -2.68 & -2.75 & -2.78 & -2.85 & -2.72 & -2.79 \\
\hline $\mathrm{Eg}$ & 2.25 & 2.25 & 2.22 & 2.21 & 2.27 & 2.29 \\
\hline \multicolumn{7}{|c|}{ PBE1PBE /6-311G(d,p) } \\
\hline Energy & -6201.90 & -6598.58 & -6598.58 & -6995.26 & -6598.58 & -6995.26 \\
\hline HOMO & -5.17 & -5.27 & -5.21 & -5.31 & -5.20 & -5.30 \\
\hline LUMO & -2.82 & -2.90 & -2.94 & -3.01 & -2.89 & -2.97 \\
\hline $\mathrm{Eg}$ & 2.35 & 2.36 & 2.26 & 2.29 & 2.30 & 2.33 \\
\hline \multicolumn{7}{|c|}{ Cam-B3LYP/6-31G } \\
\hline Energy & -6202.95 & -6599.70 & -6599.71 & -6996.46 & -6599.70 & -6996.45 \\
\hline HOMO & -6.01 & -6.08 & -6.19 & -6.30 & -6.19 & -6.31 \\
\hline LUMO & -2.11 & -2.34 & -2.25 & -2.48 & -2.18 & -2.40 \\
\hline $\mathrm{Eg}$ & 3.89 & 3.73 & 3.93 & 3.82 & 4.01 & 3.91 \\
\hline \multicolumn{7}{|c|}{ Cam-B3LYP/6-31G(d) } \\
\hline Energy & -6203.94 & -6600.79 & -6600.79 & -6997.64 & -6600.79 & -6997.63 \\
\hline HOMO & -6.09 & -6.15 & -6.12 & -6.18 & -6.02 & -6.10 \\
\hline LUMO & -1.63 & -1.75 & -1.73 & -1.85 & -1.71 & -1.80 \\
\hline $\mathrm{Eg}$ & 4.46 & 4.40 & 4.38 & 4.33 & 4.30 & 4.29 \\
\hline \multicolumn{7}{|c|}{ Cam-B3LYP/6-311G(d,p) } \\
\hline Energy & -6204.71 & -6603.07 & -6603.07 & -7000.10 & -6603.06 & -7000.10 \\
\hline HOMO & -6.53 & -5.14 & -5.12 & -5.20 & -5.04 & -5.15 \\
\hline LUMO & -1.81 & -3.04 & -3.04 & -3.14 & -3.03 & -3.12 \\
\hline $\mathrm{Eg}$ & 4.72 & 2.10 & 2.07 & 2.05 & 2.01 & 2.03 \\
\hline
\end{tabular}



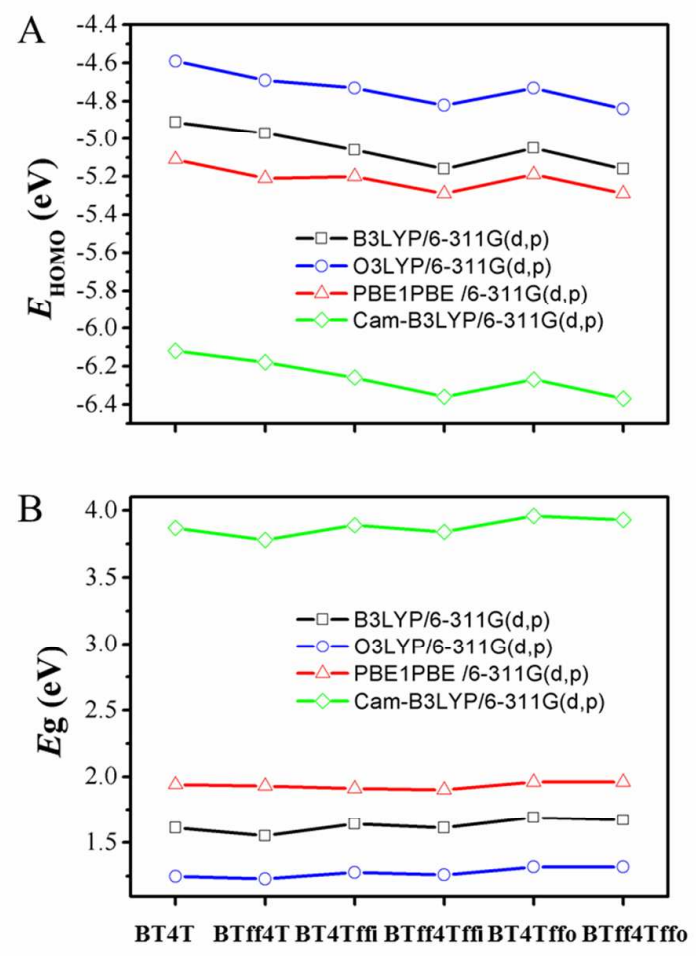

Figure S3. (A) HOMO energies and (B) band gaps of polymers with various functionals under 6-311G(d,p) basis set. 


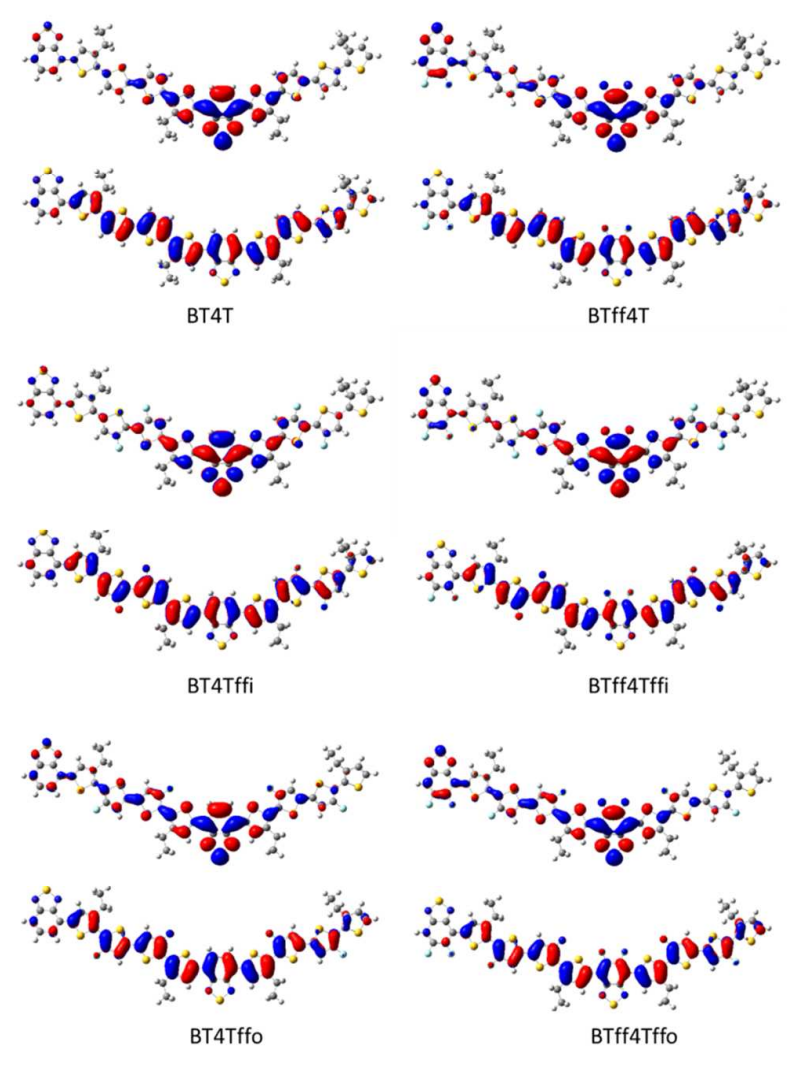

Figure S4. Frontier molecular orbital distribution of BT4T and its fluorinated derivatives.

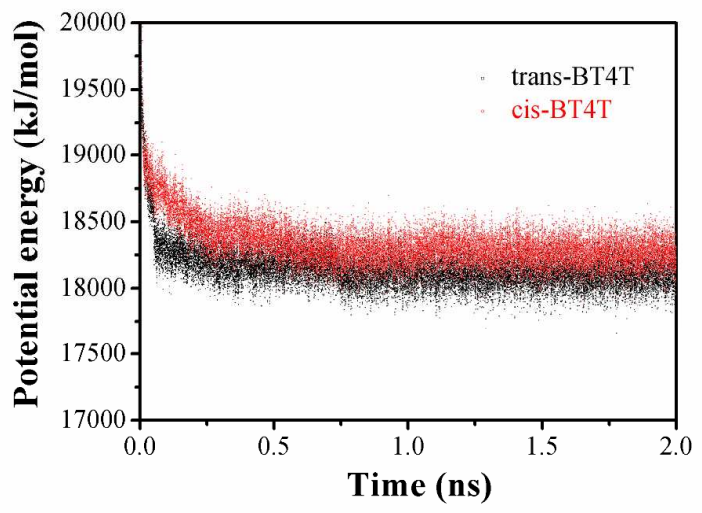

Figure S5. Potential energy as a function of time of trans- and cis-BT4T.

Table S5. Calculated reorganization energies of BT4T and its fluorinated derivatives 


\begin{tabular}{cccc}
\hline & $\lambda 1=\mathrm{E}^{*}-\mathrm{E}(\mathrm{eV})$ & $\lambda 2=\mathrm{E}_{+}{ }^{*}-\mathrm{E}_{+}(\mathrm{eV})$ & $\lambda(\mathrm{eV})$ \\
\hline BT4T & 0.134 & 0.253 & 0.387 \\
BTff4T & 0.135 & 0.263 & 0.398 \\
BT4Tffi & 0.141 & 0.198 & 0.339 \\
BTff4Tffi & 0.141 & 0.210 & 0.351 \\
BT4Tffo & 0.131 & 0.141 & 0.273 \\
BTff4Tffo & 0.133 & 0.140 & 0.273 \\
\hline
\end{tabular}

\section{References}

(1) Jheng, J.; Lai, Y.; Wu, J.; Chao, Y.; Wang, C.; Hsu, C. Influences of the Non-Covalent Interaction Strength on Reaching High Solid-State Order and Device Performance of a Low Bandgap Polymer with Axisymmetrical Structural Units. Adv. Mater. 2013, 25, 2445-2451.

(2) Chen, Z.; Cai, P.; Chen, J.; Liu, X.; Zhang, L.; Lan, L.; Peng, J.; Ma, Y.; Cao, Y. Low Band-Gap Conjugated Polymers with Strong Interchain Aggregation and Very High Hole Mobility Towards Highly Efficient Thick- Film Polymer Solar Cells. $A d v$. Mater. 2014, 26, 2586-2591.

(3) Jo, J. W.; Bae, S.; Liu, F.; Russell, T. P.; Jo, W. H. Comparison of Two D-A Type Polymers with Each Being Fluorinated on D and A Unit for High Performance Solar Cells. Adv. Funct. Mater. 2015, 25, 120-125.

(4) Jo, J. W.; Jung, J. W.; Jung, E. H.; Ahn, H.; Shin, T. J.; Jo, W. H. Fluorination on Both D and A Units in D-A Type Conjugated Copolymers Based on Difluorobithiophene and Benzothiadiazole for Highly Efficient Polymer Solar Cells. Energ. Environ. Sci. 2015, 8, 2427-2434.

(5) Hu, H.; Jiang, K.; Yang, G.; Liu, J.; Li, Z.; Lin, H.; Liu, Y.; Zhao, J.; Zhang, J.; Huang, F.; et al. Terthiophene-Based D-A Polymer with an Asymmetric Arrangement of Alkyl Chains That Enables Efficient Polymer Solar Cells. J. Am. Chem. Soc. 2015, 137, 14149-14157.

(6) Cornil, J.; Gueli, I.; Dkhissi, A.; Sancho-Garcia, J. C.; Hennebicq, E.; Calbert, J. P.; Lemaur, V.; Beljonne, D.; Bredas, J. L. Electronic and Optical Properties of Polyfluorene and Fluorene-Based Copolymers: A Quantum-Chemical Characterization. J. Chem. Phys. 2003, 118, 6615-6623.

(7) Rand, B. P.; Genoe, J.; Heremans, P.; Poortmans, J. Solar Cells Utilizing Small Molecular Weight Organic Semiconductors. Prog. Photovoltaics 2007, 15, 659-676.

(8) He, Y.; Li, Y. Fullerene Derivative Acceptors for High Performance Polymer Solar Cells. Phys. Chem. Chem. Phys. 2011, 13, 1970-1983.

(9) Scholes, G. D.; Rumbles, G. Excitons in Nanoscale Systems. Nature Mater. 2006, 5, 683-696.

(10) Winkler, M.; Houk, K. N. Nitrogen-Rich Oligoacenes: Candidates for N-Channel Organic Semiconductors. J. Am. Chem. Soc. 2007, 129, 1805-1815. 